REVISTA MATEMÁTICA COMPLUTENSE

Volumen 12, número 1: 1999

http://dx.doi.org/10.5209/rev_REMA.1999.v12.n1.17181

\title{
General stable bundles arising as normal bundles of projective curves.
}

\author{
Edoardo BALLICO and Luciana RAMELLA ${ }^{1}$
}

\begin{abstract}
Let $X$ be a smooth projective curve of genus $g \geq 2$. For all integers $r, \delta$ with $r>0$ let $M(X ; r, \delta)$ be the moduli scheme of rank $r$ stable vector bundles on $X$ with degree $\delta$.

Here we prove that for all integers $n, d$ with $n \geq 3$ and $d \geq$ $(5 n-8) g+2 n^{2}-5 n+4$ there is an open dense subset $\Omega$ of $M(X ; n-1,(n+1) d+2 g-2)$ such that for every $N \in \Omega$ there is a non-degenerate degree $d$ embedding of $X$ in $\mathbb{P}^{n}$ with $N$ as normal bundle.

For the proof we use reducible smoothable curves.
\end{abstract}

\section{Introduction}

Let $X$ be a smooth curve and $f: X \rightarrow \mathbb{P}^{n}$ an embedding. We have an exact sequence of bundles on $X: 0 \rightarrow T_{X} \rightarrow f^{*} T_{P^{n}} \rightarrow N_{f} \rightarrow 0$. The quotient $N_{f}$ is called the normal bundle of $f$. Put $C:=f(X)$. The bundle $N_{f}$ is isomorphic to the normal bundle $N_{C}$ of $C$, that is the dual bundle of $\mathcal{I}_{C} / \mathcal{I}_{C}^{2}$, where $\mathcal{I}_{C}$ denotes the ideal sheaf of $C$.

Several properties of the embedding $f$ of $X$ are reflected by the geometrical and cohomological properties of the vector bundle $N_{C}$ of $C=f(X)$. For instance the Hilbert scheme of $\mathbb{P}^{n}$ at $C$ has $H^{0}\left(C, N_{C}\right)$ as tangent space, moreover, if $h^{1}\left(C, N_{C}\right)=0$, then it is smooth at $C$ and of dimension $h^{0}\left(C, N_{C}\right)$.

\footnotetext{
'Both authors were partially supported by MURST and GNSAGA of CNR (Italy).

1991 Mathematics Subject Classification: 14H10, 14H60, $14 \mathrm{Co5}$.

Servicio Publicaciones Univ. Complutense. Madrid, 1999.
} 
Furthermore, if the homogeneous ideal of $C$ is generated by forms of degree $\leq k$, then $N_{C}^{\curlyvee}(k)$ is spanned by its global sections.

Several results on smooth curves in $\mathbb{P}^{n}$ were proved finding suitable reducible curves in $\mathbb{P}^{n}$ with "good" normal bundle.

Here we want to study the normal bundle $N_{C}$ as an abstract bundle on $C \cong X$. There is a natural question.

Which bundles on a smooth projective curve $X$ are isomorphic to bundles arising as normal bundles of some non-degenerate embedding $f$ of $X$ in $\mathbb{P}^{n}$ ?

The structure and properties of the vector bundles on $X$ depends on the genus $g$ of $X$. The cases $g=0, g=1$ and $g \geq 2$ have a very different flavour because only if $g \geq 2$ there are many stable bundles on $X$.

From now on we will assume that $X$ has genus $g \geq 2$. For all integers $r, \delta$ with $r>0$ let $M(X ; r, \delta)$ be the moduli scheme of rank $r$ stable vector bundles on $X$ with degree $\delta$. The scheme $M(X ; r, \delta)$ is an irreducible smooth variety of dimension $r^{2}(g-1)+1$ (see [9] or [10]).

In this paper we prove the following result:

Theorem 1. Fix integers $g, n, d$ with $n \geq 3, g \geq 2$ and

$$
d \geq(5 n-8) g+2 n^{2}-5 n+4 .
$$

Let $X$ be a smooth projective curve of genus $g$. Then there is a Zariski open dense subset $\Omega$ of the moduli scheme $M(X ; n-1,(n+1) d+2 g-2)$ such that for every $N \in \Omega$ there is a non-degenerate degree $d$ embedding of $X$ in $\mathbb{P}^{n}$ with $N$ as normal bundle.

We think that the lower bound on $d$ in the statement of Theorem 1 is not optimal. Let $\operatorname{Hom}_{d}^{\prime}\left(X, \mathbb{P}^{n}\right)$ be the scheme of all degree $d$ nondegenerate embeddings of $X$ into $\mathbb{P}^{n}$. The comparison of the dimension of the schemes $H_{o m}^{\prime}\left(X, \mathbb{P}^{n}\right)$ and $M(X ; n-1,(n+1) d+2 g-2)$ shows that a rather large lower bound on $d$ is necessary (say $(n+1)(d-n-$ $g)+g \geq(n-1)^{2}(g-1)+1$ for non-special embeddings).

To prove Theorem 1, we will use reducible smoothable curves $Y$ in $\mathbb{P}^{n}$ and the properties of stable bundles on reducible curves proved in [6] and [2].

We work over an algebraically closed field $\mathbf{k}$ with $\operatorname{char}(\mathbf{k})=0$. 


\section{Preliminary remarks}

Let $X$ be a smooth projective curve and $P \in X$. Consider two vector bundles $E$ and $F$ on $X$ fitting in an exact sequence

$$
0 \rightarrow E \rightarrow F \rightarrow \mathcal{O}_{P} \rightarrow 0
$$

where $\mathcal{O}_{P}$ is the skyscraper sheaf of length 1 supported by $P$. Note that we have $\operatorname{rank}(E)=\operatorname{rank}(F)$ and $\operatorname{deg}(F)=\operatorname{deg}(E)+1$.

We will say that $F$ is obtained from $E$ making a positive elementary transformation supported by $P$ and that $E$ is obtained from $F$ making a negative elementary transformation supported by $P$.

Note that a negative elementary transformation of the vector bundle $F$ supported by a point $P \in X$ corresponds to a point of the projective bundle $\mathbb{P}(F)$ belonging to the fiber $\mathbb{P}(F)_{P}$. Moreover a positive elementary transformation of the vector bundle $E$ supported by $P \in X$ corresponds to a point of $\mathbb{P}\left(E^{\vee}\right)_{P}$.

Remark 2. Fix a smooth projective curve $X$ of genus $g \geq 2$ and integers $r, \delta$ with $r>0$. It is very well-known (see for instance [3] Lemma 2.5 in arbitrary characteristic) that for a general $E \in M(X ; r, \delta)$ we have that a general negative elementary transformation of $E$ and a general positive elementary transformation of $E$ are both stable.

Since $\operatorname{char}(\mathbf{k})=0$, a strong result is true: there is a Zariski open dense subset $U$ of $M(X ; r, \delta)$ such that for every $E \in U$ every positive elementary transformation of $E$ is stable and also every negative elementary transformation of $E$ is stable (use [8] Thm. 3.10, or the stronger statement [7] Thm. 4.4, quoted in [8] Rem. 3.14).

We will not use this stronger statement, but we will use the weaker statement in the following form.

Let $\mathcal{Z}(X ; r, \delta)$ be a ramified covering of $M(X ; r, \delta)$ such that on $\mathcal{Z}(X ; r, \delta)$ there is a semi-universal family $\alpha: \mathcal{E} \rightarrow \mathcal{Z}(X ; r, \delta)$ of stable vector bundles with rank $r$ and degree $\delta . \mathcal{E}$ is called a semi-universal bundle on $\mathcal{Z}(X ; r, \delta)$.

The projective bundle $\mathbb{P}\left(\mathcal{E}^{\vee}\right) \rightarrow \mathcal{Z}(X ; r, \delta)$ parameterizes the positive elementary transformations of the vector bundles corresponding to the points of $\mathcal{Z}(X ; r, \delta)$. Set $\mathcal{Z}^{\prime}(X ; r, \delta):=\mathbb{P}\left(\mathcal{E}^{\vee}\right)$. By the first part of the Remark we obtain a rational map $\beta$ from $\mathcal{Z}^{\prime}(X ; r, \delta)$ to $M(X ; r, \delta+1)$. 
By using negative elementary transformations, from the first part of the Remark, we obtain that the above rational map is dominant.

Notation 3. The above construction may be done for flat families of smooth curves with a prescribed section.

We will call this type of construction "up to adding parameters" and we will pass very often to ramified covering to obtain semi-universal families of bundles and families of sections of flat families of curves just saying "up to a finite covering".

As in [3], §2, we consider the following concept:

Definition 4. Let $X$ be a smooth projective curve of genus $g \geq 2$ and $E$ a rank $r$ vector bundle on $X$.

Let $t(E)$ be the minimal integer $t$ such that a general $G \in$ $M(X ; r, \operatorname{deg}(E)+t)$ is obtained from $E$ by $t$ positive elementary transformations, i.e. such that $G$ has a subsheaf isomorphic to $E$. We will call $t(E)$ the generality index of $E$.

Remark 5. a) If $E$ is a rank $r$ vector bundle on $X$, from Remark 2, we have that, for every $t \geq t(E)$, a general $G \in M(X ; r, \operatorname{deg}(E)+t)$ is obtained from $E$ by $t$ (general) positive elementary transformations.

Note that for every line bundle $L \in \operatorname{Pic}(X)$ we have $t(E \otimes L)=t(E)$.

Easy examples with direct sums of line bundles show that the integer $t(E)$ depends on the instability degree of the bundle $E$.

b) Following the construction of Remark 2, we can define "up to a finite covering" the scheme $\mathcal{Z}^{(t)}(X ; r, \delta)$ parameterizing $t$ positive elementary transformations of stable bundles in $M(X ; r, \delta)$ and we have a rational map [4] $\beta: \mathcal{Z}^{(t)}(X ; r, \delta) \rightarrow M(X ; r, \delta+t)$.

If $E \in M(X ; r, \delta)$, denote by $\beta_{E}$ the restriction of the above rational map $\beta$ to the fiber of $\mathcal{Z}^{(t)}(X ; r, \delta)$ at $E$. Then, for $t \geq t(E)$, we have that the rational map $\beta_{E}$ is dominant.

c) Let $E \in M(X ; r, \delta)$, the scheme parameterizing $t$ positive elementary transformations of $E$ is of dimension $r t$. Then if the above rational map $\beta_{E}$ is dominant, we have $r t \geq r^{2}(g-1)+1$. Thus we obtain the bound $t(E) \geq r(g-1)+1$.

In [3] we proved the following results:

Lemma 6. a) ([3] Lemma 2.8) We have $t\left(r \mathcal{O}_{X}\right)=r g(r \geq 2)$.

b) ([3] Lemma 2.9) Let $E$ be a rank $r$ vector bundle on $X$ spanned by its global sections. Then $t(E) \leq(r-1) \operatorname{deg}(E)+r g$. 
Remark 7. Denote by $G(r, \nu)$ the Grassmannian of the rank $r$ quotients of $\mathbf{k}^{\nu}$. Let $X$ be a smooth projective curve of genus $g \geq 2$ and $E \in M(X ; r, \delta)$. Assume $\delta>2 g r+r$. Since $h^{1}\left(X, E\left(-P-P^{\prime}\right)\right)=$ $h^{1}(X ; E(-2 P))=0$ for all $P, P^{\prime} \in X([9]$ Lemma 5.2), we have that $E$ is spanned by its global sections and the quotient $\mathcal{O}_{X} \otimes H^{0}(X, E) \rightarrow$ $E \rightarrow 0$ induces an embedding $\varphi: X \rightarrow G(r, \nu)$, where $\nu=h^{0}(X, E)=$ $\delta+r(1-g)$.

\section{Proof of Theorem 1}

We describe the proof in several steps.

Step 1. We describe the smoothable nodal curves we use.

Let $\operatorname{Hom}_{d}^{\prime}\left(X, \mathbb{P}^{n}\right)$ be the scheme parameterizing the degree $d$ nondegenerate embeddings of $X$ in $\mathbb{P}^{n}$. It gives a subscheme $\stackrel{\circ}{H}$ of the Hilbert scheme [4] ilb $_{d, g}\left(\mathbb{P}^{n}\right)$ parameterizing the degree $d$ genus $g$ curves in $\mathbb{P}^{n}$. The scheme $\stackrel{\circ}{H}$ is irreducible and, since $d>2 g-2$, we have $\stackrel{\circ}{\mathrm{H} C H} \mathrm{Hilb}_{d, g}\left(\mathbb{P}^{n}\right)_{\text {reg }}$.

Let $\boldsymbol{H}$ be the open subset of the closure of $\stackrel{\circ}{\boldsymbol{H}}$ in $H i l b_{d_{r} g}\left(\mathbb{P}^{n}\right)$ formed by reduced curves with only nodes as singularities.

Fix a very ample line bundle $L \in P i c^{n+g}(X)$ with $h^{1}(X, L)=0$. Let $h: X \rightarrow \mathbb{P}^{m}:=\mathbb{P}\left(H^{0}(X, L)\right)$ be the corresponding linearly normal embedding.

Set $Y:=h(X)$ and denote by $N:=N_{h}=N_{Y}$ its normal bundle. We have $\operatorname{deg}(N(-1))=2(n+g)+2 g-2$. Since $N(-1)$ is spanned by its global sections, by Lemma 6 , we have $t(N(-1)) \leq(n-2)(4 g+2 n-$ $2)+(n-1) g$. Hence $t(N)=t(N(-1)) \leq(5 n-9) g+(n-2)(2 n-2)$.

Note that $d-n-g \geq t(N)$. Fix $t:=d-n-g$ general points $P_{i}$ of $Y$ and for every point $P_{i}$ a general line $D_{i}$ passing through it, $1 \leq i \leq t$.

Set $Z:=Y \cup\left(\cup_{i=1}^{i} D_{i}\right)$. By [11] (or see [1] Thm.0, for a stronger statement), $Z$ is smoothable to a curve $C$ given by a degree $d$ embedding of $X$ in $\mathbb{P}^{n}$, i.e. $Z \in \boldsymbol{H}$.

We call $\mathcal{S}_{Y}$ the subscheme of $\boldsymbol{H}$ parameterizing all such curves $Z$ with fixed $Y$ and varying the points $P_{i}$ and the lines $D_{i}, 1 \leq i \leq t$, with the only restrictions that for every $i$ we have $P_{i} \in D_{i}, D_{i} \cap Y=\left\{P_{i}\right\}$, $D_{i}$ intersects quasi-transversally $Y$ and $D_{i} \cap D_{j}=\emptyset$ for $i \neq j$. These 
restrictions are equivalent to the fact that $Z$ is of arithmetic genus $g$.

Denote by $\mathcal{G}(1, n)$ the Grassmannian of the lines in $\mathbb{P}^{n}$ and by $\mathcal{G}_{Y}(1, n)$ the subvariety of $\mathcal{G}(1, n)$ formed by the lines of $\mathbb{P}^{n}$ meeting $Y$.

The scheme $\mathcal{S}_{Y}$ is isomorphic to an open subset of the symmetric $t$-product $S^{(t)}\left(\mathcal{G}_{Y}(1, n)\right)$ of $\mathcal{G}_{Y}(1, n)$.

If $Z$ is a nodal curve as above, the bundle $N_{Z \mid Y}$ is obtained from the normal bundle $N$ of $Y$ making $t$ positive elementary transformations supported by the points $P_{i}, 1 \leq i \leq t$ and, for every $i$, uniquely determined by the plane defined by $D_{i}$ and the tangent line of $Y$ at $P_{i}$ ([5] Cor. 3.2 and its proof for the case $n>3$ ).

Since $t=d-n-g \geq t(N)$, varying the general points $P_{i}$ of $Y$ and the lines $D_{i}$ with $P_{i} \in D_{i}$ for all $i$, "up to a finite covering" the associated bundles $N_{Z \mid Y}$ cover a Zariski open dense subset of $M(X ; n-1,(n+$ 1) $(n+g)+2 g-2+t$ ) (see Remark 5).

Note that, for every $i, N_{Z \mid D_{i}}$ is a general positive elementary transformation of $N_{D_{i}} \cong(n-1) \mathcal{O}_{P_{1}}$ (1) ([5] Cor 3.2 and its proofs for the case $n>3$ ), then $N_{Z \mid D_{i}} \cong \mathcal{O}_{P^{1}}(2) \oplus(n-2) \mathcal{O}_{P^{1}}$ (1) (if $F$ is a vector bundle and $s$ is a positive integer, we denote by $s F$ the direct sum of $s$ copies of $F$ ).

Step 2. We consider auxiliary bundles associated to the normal bundle. Consider the above reducible curve $Z$. For all integers $i$ with $1 \leq i \leq t$, take $Q_{i} \in D_{i}, Q_{i} \neq P_{i}$ and the bundle $N_{Z}^{\prime}$ on $Z$ obtained by $N_{Z}$ making a general negative elementary transformation supported by the points $Q_{1}, \ldots, Q_{t}$. We have an exact sequence of sheaves on $Z$

$$
0 \rightarrow N_{Z}^{\prime} \rightarrow N_{Z} \stackrel{\eta}{\rightarrow} \mathcal{O}_{\left\{Q_{1}, \ldots, Q_{t}\right\}} \rightarrow 0 .
$$

Note that $Q_{i} \in Z_{\text {reg }}$, then we may define a negative elementary transformation supported by $Q_{i}$ of a bundle on the singular curve $Z$. The sheaf $N_{Z}^{\prime}$ is locally free and such that $N_{Z \mid Y}^{\prime} \cong N_{Z \mid Y}$, because the points $Q_{1}, \ldots, Q_{1}$ do not belong to $Y, N_{Z \mid D_{i}}^{\prime} \simeq(n-1) O_{p},(1)$, for $1 \leq i \leq t$, because $\eta$ is general.

Now set $N_{Z}^{\prime \prime}:=N_{Z}^{\prime}\left(-Q_{1}-\cdots-Q_{t}\right)$. We have that $N_{Z}^{\prime \prime}$ is a locally free sheaf on $Z$, with $N_{Z D_{i}}^{\prime \prime} \cong(n-1) \mathcal{O}_{p 1}$, for every $1 \leq i \leq t_{\text {, }}$ and $N_{Z \mid Y}^{\prime \prime} \cong N_{Z \mid Y}$.

We claim that $N_{Z}^{\prime \prime}$ is spanned by its global sections and that 
$h^{1}\left(Z, N_{Z}^{\prime \prime}\right)=0$. To check the claim consider the following Mayer-Vietoris exact sequence:

$$
0 \rightarrow N_{Z}^{\prime \prime} \rightarrow N_{Z \mid Y}^{\prime \prime} \oplus\left(\oplus_{i=1}^{t} N_{Z \mid D_{i}}^{\prime \prime}\right) \rightarrow \oplus_{i=1}^{i} N_{Z \mid P_{i}}^{\prime \prime} \rightarrow 0 .
$$

The triviality of $N_{Z \mid D_{i}}^{\prime \prime}$, for every $1 \leq i \leq t$, implies the surjectivity of the last map of global sections induced by the above exact sequence. Moreover $N_{Z \mid Y}^{\prime \prime}$ is spanned, thus $N_{Z}^{\prime \prime}$ is spanned.

Step 3. We construct a flat family of stable auxiliary bundles.

Let $\mathcal{C}$ be the universal curve of $\boldsymbol{H}$ (see Step 1). $\mathcal{C}$ is a subscheme of $\mathbb{P}_{\boldsymbol{H}}^{n}$ and we can consider the normal bundle $N_{\mathcal{C} / \boldsymbol{P}_{H}^{n}}$, that is $\boldsymbol{H}$-flat (the curves in $\boldsymbol{H}$ are locally complete intersection).

If $C$ is a curve of $\boldsymbol{H}$, a negative elementary transformation of $N_{C}$ is given by a k-point of the projective bundle $\mathbb{P}\left(N_{C}\right)$, i.e. by a quotient $N_{C} \rightarrow \mathbf{k} \rightarrow 0$.

The scheme $\boldsymbol{E}$ parameterizing the negative elementary transformations of the normal bundle of curves in $\boldsymbol{H}$ is given by the projective bundle $\mathbb{P}\left(N_{\mathcal{C} / \boldsymbol{P}_{\boldsymbol{H}}^{n}}\right)$.

If $\boldsymbol{Y}$ is a $\boldsymbol{X}$-scheme, we denote by $S_{\boldsymbol{X}}^{(t)}(\boldsymbol{Y})$ the symmetric $t$-product of $\boldsymbol{Y}$ on $\boldsymbol{X}$.

Moreover, we denote by $H i l b_{t}(\boldsymbol{Y} / \mathbf{X})$ the relative Hilbert scheme of $t$ points of $Y$.

Let $\boldsymbol{T}$ be the scheme parameterizing the curves $C$ of $\boldsymbol{H}$ with $t$ assigned distinct points. $\boldsymbol{T}$ is an open irreducible subscheme of the relative Hilbert scheme $\mathrm{Hilb}_{t}(\mathrm{C} / \mathrm{H})$ and it is isomorphic to an open subscheme of the symmetric product $S_{H}^{(t)}(\mathcal{C})$.

Let $\mathcal{U}$ be the scheme parameterizing the above curves $Z$ having an assigned point $Q_{i}$ on each line $D_{i}$, with $Q_{i} \neq P_{i}$. The scheme $U_{Z}$ parameterizing the above configurations with fixed $Y$ is isomorphic to an open subscheme of the symmetric product $S^{(t)}\left(\Omega_{Y}(1, n)\right)$, where $\Omega_{Y}(1, n)$ is the universal scheme over $\mathcal{G}_{Y}(1, n)$. Note that $\mathcal{U}$ is an open subscheme of an irreducible component of the boundary $\boldsymbol{T} \backslash \mathcal{W}$, where $\mathcal{W}$ is the open subscheme of $\boldsymbol{T}$ consisting of smooth curves.

Now let $\boldsymbol{T}^{\prime}$ be the scheme parameterizing the curves $C$ of $\boldsymbol{H}$ with $t$ assigned (smooth) distinct points $Q_{1}, \ldots, Q_{t}$ and with a negative elementary transformation of $N_{C}$ supported by $Q_{1}, \ldots Q_{t}$. 
$\boldsymbol{T}^{\prime}$ is an open irreducible subscheme of the relative Hilbert scheme $H_{i l b}(\mathbb{E} / \mathbb{H})$ and it is isomorphic to an open subscheme of the symmetric product $S_{\boldsymbol{H}}^{(t)}(\mathbb{E})$.

We have a $\boldsymbol{H}$-morphism $\tau: \boldsymbol{T}^{\prime} \rightarrow \boldsymbol{T}$.

For every point $\left(C, Q_{1}, \ldots, Q_{t}, \eta\right)$ of $\boldsymbol{T}^{\prime}$, it is well-defined a locally free subsheaf $N_{C}^{\prime \prime}$ of $N_{C}$ fitting in an exact sequence

$$
0 \rightarrow N_{C}^{\prime \prime} \rightarrow N_{C}\left(-Q_{1}-\cdots-Q_{t}\right) \stackrel{\eta}{\rightarrow} \oplus_{i=1}^{t} \mathcal{O}_{Q_{i}} \rightarrow 0 .
$$

We have $\operatorname{rank}\left(N_{C}^{\prime \prime}\right)=n-1$ and $\operatorname{deg}\left(N_{C}^{\prime \prime}\right)=\operatorname{deg}\left(N_{Z}^{\prime \prime}\right)=\operatorname{deg}\left(N_{Z \mid Y}\right)$.

We claim that for a general point of $\boldsymbol{T}^{\prime}$, the bundle $N_{C}^{\prime \prime}$ is stable.

It is sufficient to prove the claim for a general $\left(Z, Q_{1}, \ldots, Q_{t}, \eta\right) \in$ $\tau^{-1}(\mathcal{U})$ (see [6] Thm. 2.4). We have that $N_{Z \mid Y}^{\prime \prime} \cong N_{Z \mid Y}$ is stable and that for every line $D_{i}$ the bundle $N_{Z \mid D_{i}}^{\prime \prime}$ is trivial and hence semistable. Then $N_{Z}^{\prime \prime}$ is a stable bundle on $Z$ (see [2] Lemma 1.1).

Step 4. We construct morphisms in a Grassmannian.

By Remark 7, the above bundle $N_{C}^{\prime \prime}$ is spanned by its global sections, we have $h^{1}\left(C, N_{C}^{\prime \prime}\right)=0$ and $N_{C}^{\prime \prime}$ gives an embedding $X \cong C \rightarrow G(n-1, \nu)$, where $\nu=h^{0}\left(C, N_{C}^{\prime \prime}\right)=d+3 g+n^{2}+n-3$.

Note that $N_{Z}^{\prime \prime}$ is spanned by its global sections (Step 2) and $H^{0}\left(Z, N_{Z}^{\prime \prime}\right)$ induces a morphism of $Z$ in $G(n-1, \nu)$ which embeds $Y$ and contracts the lines $D_{i}$ to the images of the points $P_{i}$.

Hence we have also a flat family of morphisms from $X$ into the Grassmannian $G(n-1, \nu)$ (we have $\nu=h^{0}\left(Z, N_{Z}^{\prime \prime}\right)=h^{0}\left(Y, N_{Z}^{\prime \prime} \mid Y\right)=$ $h^{0}\left(C, N_{C}^{\prime \prime}\right)$ ), such that the image of all curves are all isomorphic to $X$ and the bundles $N_{Z \mid Y}^{\prime \prime}$ and $N_{C}^{\prime \prime}$ arise as restriction of the universal quotient bundle of $G(n-1, \nu)$.

Step 5. We conclude.

Since, "up to a finite covering", varying $Z$ in $S_{Y}$ we obtain as $N_{Z \mid Y}^{\prime \prime}$ a Zariski dense open subset of $M(X ; n-1, \delta)$, where $\delta=(n+2) g+d+n^{2}-2$ (Step 1), we have that the bundles $N_{C}^{\prime \prime}$ cover a Zariski dense open subset of $M(X ; n-1, \delta)$.

Theorem 1 follows from Remark 2 and the way we obtained $N_{C}^{\prime \prime}$ from $N_{C}$. 


\section{References}

[1] E. Ballico, Ph. Ellia, On the postulation of a general projection of a curve in $\mathbb{P}^{n}, n \geq 4$, Annali Mat. Pura Appl. (IV) 147 (1987), $267-301$

[2] E. Ballico, G. Hein, On the stability of the restriction of $T_{P^{n}}$ to projective curves, to appear in Archiv. der Math.

[3] E. Ballico, L. Ramella, The restricted tangent bundle of smooth curves in Grassmannians and curves in flag varieties, preprint

[4] R. Hartshorne, Algebraic Geometry, Springer-Verlag, New YorkHeidelberg-Berlin, 1977

[5] R. Hartshorne, A. Hirschowitz, Smoothing algebraic space curves, in "Algebraic geometry Sitges $1983^{n}$, L.N.in Math.n1124, SpringerVerlag 1984, 98-131

[6] G. Hein, H. Kurke, Restricted tangent bundle of space curves, Israel Math. Conf. Proc. 9 (1996), 283-294

[7] A. Hirschowitz, Problèmes de Brill-Noether en rang supérieur, unpublished preprint

[8] H. Lange, Some geometrical aspects of vector bundles on curves, Aportaciones Matemáticas 5 (1992), 53-74

[9] P. E. Newstead, Introduction to moduli problems and orbit spaces, Tata Institute Lectures on Mathematics 51, 1978

[10] C. S. Seshadri, Fibrés vectoriels sur les courbes algébriques (rédigé par J.M. Drezet), Astérisque n. 96, Soc. Math. de France, 1982

[11] A. Tannebaum, Deformation of space curves, Arch. Math. 34 $(1980), 37-42$ 
E. Ballico

Department of Mathematics

University of Trento

38050 Povo (TN)

Italy

e-mail: ballicoescience.unitn. it

fax: (39) 0461881624

L. Ramella

Department of Mathematics

University of Genova

v. Dodecaneso 35

16146 Genova

Italy

$e$-mail: ramellaodima.unige.it

Recibido: 9 de Septiembre de 1998 